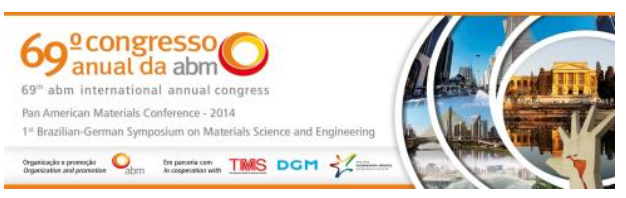

Tema: Nanotecnologia

\title{
CARACTERIZAÇÃO MICROESTRUTURAL E DA TEXTURA CRISTALORÁFICA DA LIGA AL AA 1070 PRENSADA EM CANAIS EQUIANGULARES A FRIO VIA ROTA A*
}

\author{
Rachel Santos Mendes ${ }^{1}$ \\ Christien Guisard Hauegen² \\ Manuela Fontana ${ }^{3}$ \\ Gabriel Gonçalves Pessoa de Castro ${ }^{4}$ \\ Jefferson Fabrício Cardoso Lins $^{5}$
}

\section{Resumo}

O objetivo deste trabalho foi analisar o refinamento da liga AI AA1070 deformada via ECAP (Equal Channel Angular Pressing) pela rota A em dois passes consecutivos. $O$ material foi prensado a frio e a deformação verdadeira acumulada foi de 2,38 . A caracterização microestrutural foi realizada com o auxílio da técnica de MEV. E a evolução da textura foi analisada através da técnica EBSD. A avaliação do comportamento mecânico da amostra foi realizada através do ensaio de microdureza de Vickers. Os resultados mostraram uma intensa redução no tamanho de grão após o $1^{\circ}$ passe, seguido de um decréscimo considerável no $2^{\circ}$ passe. A

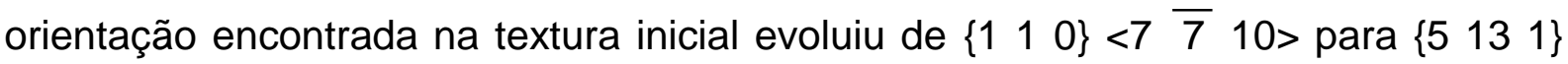
<16 $\overline{7} 11>$ após o $2^{\circ}$ passe. O comportamento mecânico apresentou um estado estacionário entre $01^{\circ}$ e $2^{\circ}$ passe devido à ocorrência do fenômeno de recuperação dinâmica. Concluiu-se que um refinamento considerável foi obtido.

Palavras-chave: ECAP; Alumínio; Microestrutura; Textura cristalográfica.

\section{MICROSTRUCTURAL AND CRYSTALLOGRAPHIC TEXTURE CHARACTARIZATION OF AA1070 AL ALLOY DEFORMED BY EQUAL CHANNEL ANGULAR PRESSING VIA ROUTE A}

\section{Abstract}

The objective of this study was to analyze the refinement of AA1070 Al alloy deformed via ECAP by route $A$ in two consecutive passes. The material was coldpressed and the true strain accumulated was 2.38. The microstructural characterization was performed with the aid of SEM technique. The evolution of texture was analyzed by EBSD technique. The evaluation of the mechanical behavior was performed using the Vickers hardness test. The results showed a marked decrease in grain size after the 1st pass, followed by a considerable decrease in the 2 nd pass. The orientation found in the initial texture has evolved from $\left\{\begin{array}{lll}1 & 1 & 0\end{array}\right\}<7 \overline{7}$ $10>$ to $\left\{\begin{array}{lll}5 & 13 & 1\end{array}\right\}<16 \overline{7} 11>$ after the 2 nd pass. The mechanical behavior showed a steady state between the 1st and 2nd pass due to the occurrence of the phenomenon of dynamic recovery. It was concluded that a considerable refinement was obtained

Keywords: ECAP; Aluminium; Microstructure; Crystallographic texture.

\footnotetext{
Eng. de Produção, Mestranda, Programa de Pós-graduação em Engenharia Metalúrgica PPGEM, Universidade Federal Fluminense (UFF), Volta Redonda, RJ, Brasil.

Eng. Metalúrgico, Mestrando, PPGEM, UFF, Volta Redonda, RJ, Brasil.

Eng. Metalúrgica, M.Sc., Doutoranda, PPGEM, UFF, Volta Redonda, RJ, Brasil.

Eng. Mecânico, Mestrando, PPGEM, UFF, Volta Redonda, RJ, Brasil.

Eng. Químico, D.Sc., Coordenador PPGEM, UFF, Volta Redonda, RJ, Brasil.
}

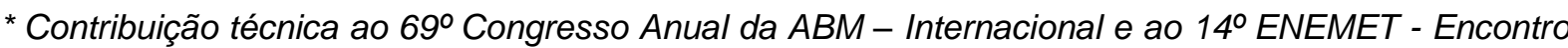
Nacional de Estudantes de Engenharia Metalúrgica, de Materiais e de Minas, 21 a 25 de julho de 2014, São Paulo, SP, Brasil.
} 


\section{INTRODUÇÃO}

A técnica de ECAP consiste em forçar um tarugo a escoar entre dois canais que possuem seções transversais iguais, contidos no interior de uma matriz rígida. À medida que o material passa por dentro dos canais, este sofre deformação plástica de natureza cisalhante simples desenvolvendo uma nova microestrutura e textura cristalográfica. Além de uma combinação singular de propriedades físicas e mecânicas. A principal vantagem do processo de ECAP é que o material pode ser deformado sem que haja a diminuição de sua seção transversal, além de melhorar as propriedades mecânicas, produzir grãos extremamente finos e uma variedade de texturas. Este processo pode ser conduzido por quatro rotas: $A, B_{A}, B_{c}$ e $C$. A rota $A$ não envolve nenhuma rotação da amostra entre os passes de deformação. A rota $\mathrm{B}_{\mathrm{A}}$, por sua vez, envolve rotações alternativas de $90^{\circ}$, nos sentidos horário e antihorário em relação à direção de prensagem. A rota $B c$ envolve rotações de $90^{\circ}$ no mesmo sentido entre cada prensagem. E por fim, a rota $C$ envolve rotações de $180^{\circ}$ entre cada passe [1]. Uma vez que a área da seção transversal se mantém inalterada é possível realizar repetidas deformações até que estas se acumulem em altos níveis. Esta repetição permite que sistemas de deslizamento sejam ativados em cada passe através da rotação da amostra nos diferentes ângulos associados a cada rota [2]. Kim e seus colaboradores [3] compararam a evolução microestrutural e sua influência nas propriedades mecânicas do alumínio comercialmente puro $(99,5 \%)$ processado via rotas A e C. Observaram que o tamanho do grão da amostra foi reduzido drasticamente após o primeiro passe de deformação e seguido de um decréscimo gradual no segundo passe. Puderam concluir que, a rota de processamento exerce maior influência no formato final dos grãos do que no tamanho médio dos mesmos. Notaram também que o limite de escoamento da deformação cisalhante aumenta com a diminuição do tamanho do grão, porém esse aumento só é significativo após o primeiro passe. A partir do segundo passe esse crescimento é gradual, o que permitiu concluir que o limite de escoamento da deformação cisalhante do alumínio comercialmente puro após dois passes de deformação é quatro vezes maior comparado ao valor do material de partida.

O contrário foi observado por Cao et al [4] que investigaram o mesmo material prensado via mesmas rotas e concluíram que somente a partir do décimo passe uma quantidade significativa de grãos ultrafinos é notada. E que mesmo após 10 passes, heterogeneidades significativas são encontradas em regiões da microestrutura em que não houve refinamento dos grãos. Conclui-se que a grande questão a ser estudada é quanto ao efeito de cada rota no material assim como o número de passes necessário para produzir uma microestrutura ultrafina e homogênea. Neste contexto, o objetivo deste trabalho é analisar o refinamento de um material grosseiro submetido ao ECAP através da rota $A$ em dois passes de deformação. O processo de deformação foi a frio e a deformação verdadeira acumulada alcançou $\varepsilon_{2}=2,38$. A investigação do refinamento da liga Al AA1070 consistiu na caracterização microestrutural, da textura cristalográfica e de seu comportamento mecânico. A caracterização microestrutural foi realizada com 0 auxílio da técnica de microscopia eletrônica de varredura (MEV). E a evolução da textura foi analisada através da técnica de difração de elétrons retroespalhados (EBSD). A avaliação do comportamento mecânico da amostra foi realizada através do ensaio de microdureza de Vickers, a fim de quantificar a energia armazenada no material após cada passe de deformação.

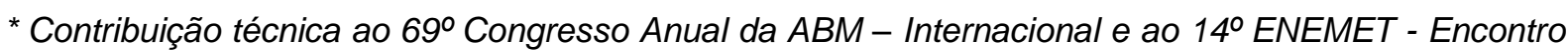
Nacional de Estudantes de Engenharia Metalúrgica, de Materiais e de Minas, 21 a 25 de julho de 2014, São Paulo, SP, Brasil.
} 


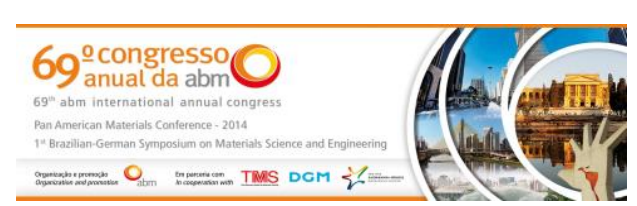

\section{MATERIAIS E MÉTODOS}

As amostras são provenientes de uma liga Al AA 1070, em forma de uma placa fornecida pela empresa Novelis do Brasil Ltda. A Tabela 1 mostra a composição química nominal da placa de Al AA1070 que será utilizada no presente trabalho. Uma chapa foi lingotada para a confecção da placa de $610 \mathrm{~mm}$ de espessura e em seguida, desbastada via laminação a quente até a espessura de $32 \mathrm{~mm}$ e sua temperatura de saída foi estimada como sendo acima de $380^{\circ} \mathrm{C}$.

Tabela 1: Composição química liga de alumínio AA1070 (teores expressos em \% em peso).

\begin{tabular}{ccccccccc}
\hline $\mathrm{Mn}$ & $\mathrm{Mg}$ & $\mathrm{Si}$ & $\mathrm{Pb}$ & $\mathrm{Fe}$ & $\mathrm{Ti}$ & $\mathrm{Cu}$ & $\mathrm{Ga}$ & $\mathrm{Al}$ \\
\hline- & - & 0,07 & 0,002 & 0,18 & 0,02 & - & 0,001 & 99,72 \\
\hline
\end{tabular}

Para a realização do processo de ECAP foi utilizada uma matriz bipartida de aço ferramenta $\mathrm{H} 13$ com dois canais idênticos com dimensões de $10 \times 10 \mathrm{~mm}$ formando um ângulo $(\Phi)$ igual a $90^{\circ}$ entre si. Antes da deformação, o canal e o tarugo foram lubrificados com uma graxa a base de sulfonato de cálcio. O punção de aço prata ao tungstênio foi fixado em uma máquina de ensaios universal EMIC DL-60 com capacidade máxima de carga de $600 \mathrm{KN}$. Os tarugos, de dimensões de $10 \times 10 \times 70$ $\mathrm{mm}$, foram prensados à velocidade de até $5 \mathrm{~mm} / \mathrm{s}$ através da rota $A$ em dois passes de deformação, à temperatura ambiente. A deformação acumulada alcançou o valor de $\varepsilon_{2}=2,38$. O raio de adoçamento dos canais é de $5 \mathrm{~mm}\left(R \approx 37^{\circ}\right)$ a fim de facilitar o fluxo do tarugo entre os canais e reduzir a carga de prensagem. A Figura 1 apresenta de maneira esquemática a parte da matriz em que ocorre interseção entre os dois canais com a presença de um raio de adoçamento [5].

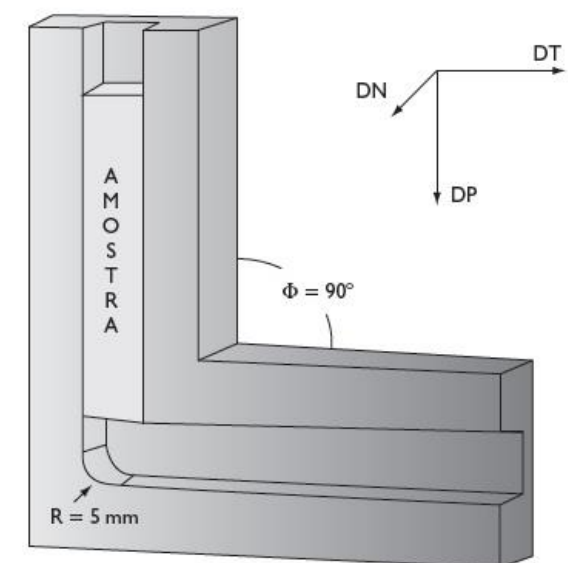

Figura 1: Desenho esquemático de uma seção da matriz ECAP bipartida mostrando o ângulo entre os canais $(\Phi)$ e raio de adoçamento $(R)$. DP - diração de prensagem, DN - direção normal e DT direção transversal. [5]

As amostras dos tarugos foram cortadas com dimensões aproximadas de $10 \times 10 \times 05 \mathrm{~mm}$, em uma cortadeira de baixa velocidade com disco diamantado. As seguintes granulometrias de $\mathrm{SiC}, 1000,1200$ e 2400 foram utilizadas no posterior lixamento das amostras. Por fim, o polimento foi realizado no equipamento ElectroMetß 4 da Buehler, empregando-se uma solução composta por 59 partes de $\mathrm{CH}_{4} \mathrm{O}$ (metanol), 35 partes de $\mathrm{C}_{2} \mathrm{H}_{4}(\mathrm{OH})_{2}$ (etileno-glicol) e 6 partes de $\mathrm{HClO}_{4}$ (ácido perclórico) (em volume). A diferença de potencial aplicada foi de $80 \mathrm{~V}$ por $90 \mathrm{~s}$, à temperatura média de $21^{\circ} \mathrm{C}$.

\footnotetext{
* Contribuição técnica ao $69^{\circ}$ Congresso Anual da ABM - Internacional e ao 14ํㅡㄹ ENEMET - Encontro Nacional de Estudantes de Engenharia Metalúrgica, de Materiais e de Minas, 21 a 25 de julho de 2014, São Paulo, SP, Brasil.
} 
(a)

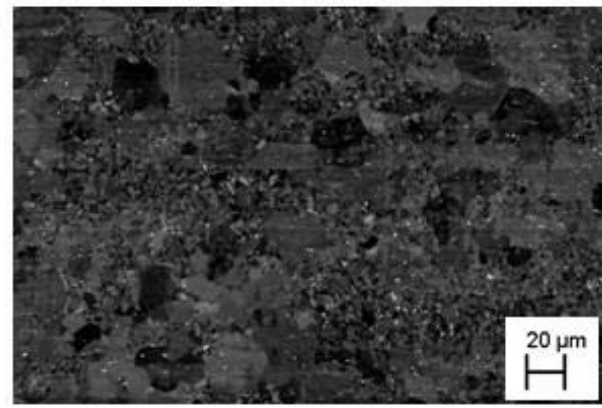

(b)

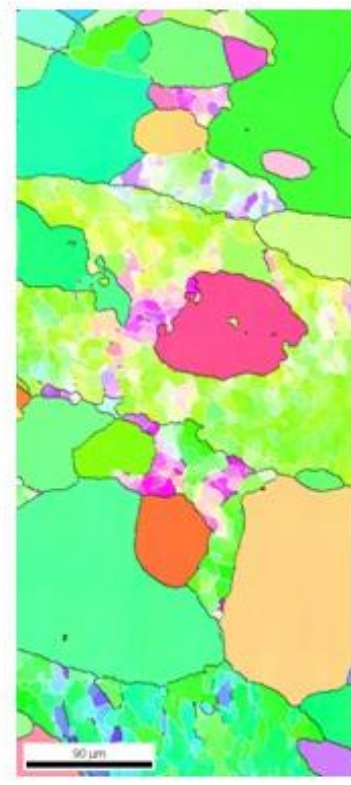<smiles>C=CC=[N+]</smiles>
DN
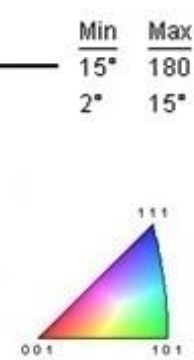

Figura 2: a) Micrografia da seção transversal do material de partida. MEV - imagem no modo de elétrons retroespalhados, $10 \mathrm{kV}$.; (b) Mapa de orientação referente à seção transversal do material de partida. $(M E V-E B S D=20 k V)$. DN e DT referem-se às direções normal e transversal respectivamente.

Para caracterização do material após o primeiro passe de deformação foi analisada uma área com um tamanho de 165,31 x 155,17 $\left(\mu^{2}{ }^{2}\right)$ e com um step size de $1 \mu \mathrm{m}$. A micrografia e o mapa de orientação do material após o primeiro passe de deformação podem ser vistas na Figura 3.

(a)

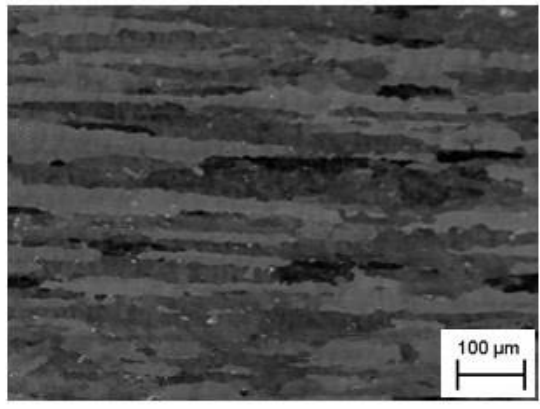

(b)

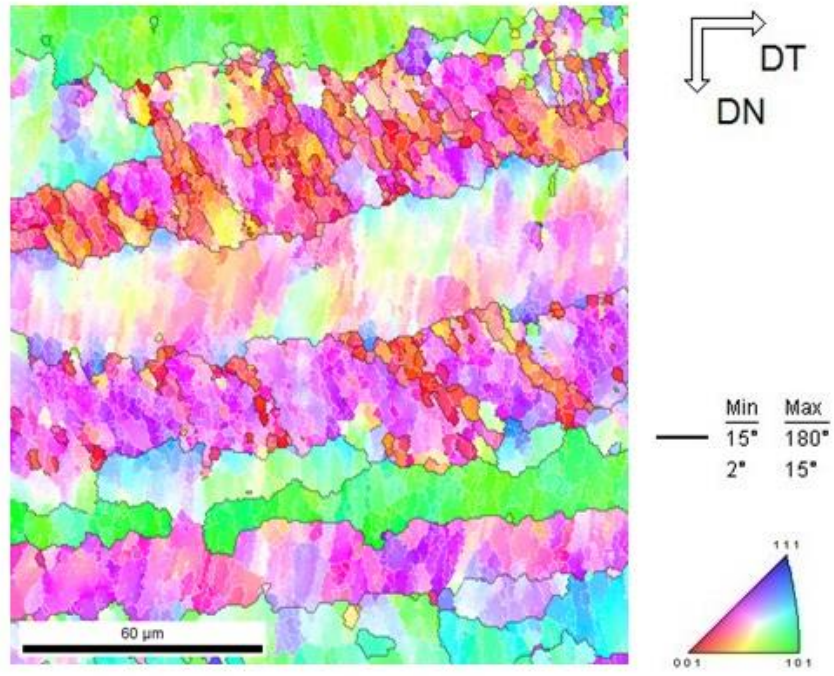

Figura 3: (a) Micrografia da seção transversal da amostra do tarugo após o primeiro passe. MEV imagem no modo de elétrons retroespalhados, 10kV.; (b) Mapa de orientação referente à seção transversal da amostra do tarugo após o primeiro passe. (MEV-EBSD $=20 \mathrm{kV})$. DN e DT referem-se às direções normal e transversal respectivamente.

A micrografia apresentada na Figura 3(a) permite verificar a existência de componentes com diferentes orientações. Ademais os contornos de grãos aparentam estar paralelos à direção transversal. Porém na figura 3(b) é possível notar que estes contornos apresentam protusões. Essa característica pode ser explicada devido ao fato de os grãos vizinhos apresentarem orientações iniciais diferentes e estes ao passarem pela zona de cisalhamento sofrem rotações. Assim,

* Contribuição técnica ao 69 Congresso Anual da ABM - Internacional e ao 14ํㅡㄹ ENEMET - Encontro Nacional de Estudantes de Engenharia Metalúrgica, de Materiais e de Minas, 21 a 25 de julho de 2014, São Paulo, SP, Brasil. 


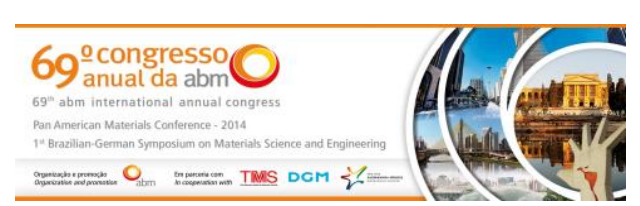

geram-se essas protusões a fim de acomodar a deformação. A comparação das Figuras 2(b), 3(b) permitiu notar de maneira instantânea uma redução no tamanho do grão. Após o primeiro passe de deformação, o grão sofreu uma redução significativa em relação ao seu tamanho médio para 5,22 $\pm 5,07 \mu \mathrm{m}$, o que caracteriza uma redução de aproximadamente $70 \%$ no tamanho médio inicial do grão. O material submetido ao primeiro passe de deformação apresentou uma microestrutura composta, principalmente, de uma estrutura bandeada constituída de grãos alongados. Nota-se ainda que, alguns desses grãos apresentam uma distribuição de cores que caracteriza a presença de subestruturas, com caráter de baixo ângulo, gerados pelo processo de subdivisão. Para este passe o material apresentou uma fração de contornos de baixo ângulo correspondente a $74 \%$. A Figura 4 apresenta a micrografia e o mapa de orientação do material após o segundo passe de deformação. A área analisada foi de 137,90 x 100,13 ( $\left.\mu^{2}{ }^{2}\right)$ com um step size de 0,5 $\mu \mathrm{m}$.

(a)
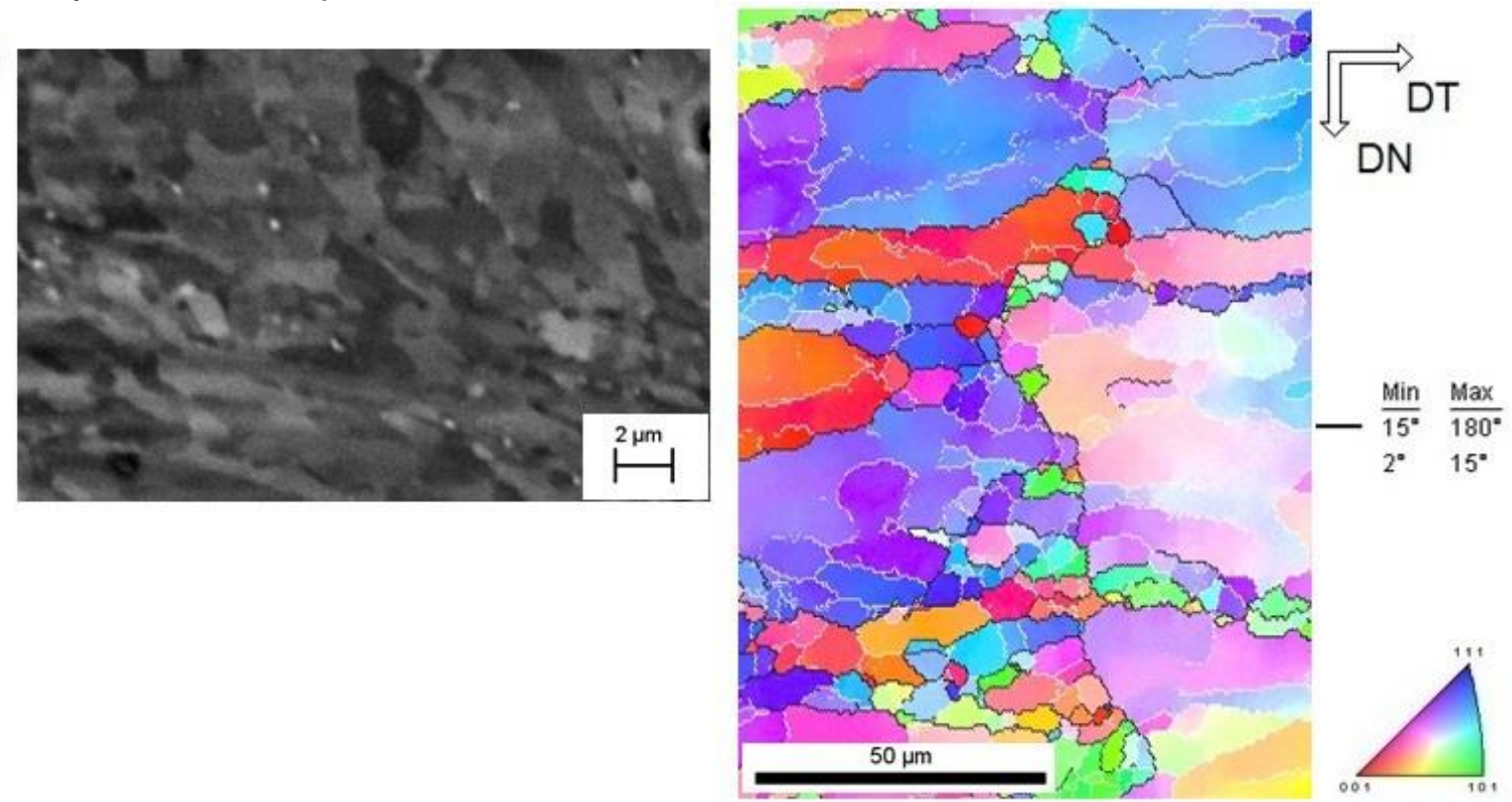

Figura 4: (a) Micrografia da seção transversal da amostra do tarugo após o segundo passe. MEV imagem no modo de elétrons retroespalhados, 10kV.; (b) Mapa de orientação referente à seção transversal da amostra do tarugo após o segundo passe. (MEV-EBSD $=15 \mathrm{kV}$ ). DN e DT referem-se às direções normal e transversal respectivamente

Para o material submetido ao segundo passe de deformação, o refinamento não foi tão proeminente. O material apresentou tamanho médio de grão de 3,40 $\pm 3,81 \mu \mathrm{m}$, o que representa uma redução a mais de $35 \%$. Além disso, foi notável a mudança no formato dos grãos. Uma microestrutura composta por grãos alongados continuou a ser observada no material submetido ao segundo passe de deformação, porém com uma maior quantidade de subgrãos. Ademais, foi possível observar um aumento na fração de contornos de alto ângulo, para 49\%. Essas mudanças em relação à quantidade de subestruturas e subgrãos estão previstas na literatura segundo a evolução da microestrutura durante a deformação, que relata a ocorrência de mudanças sucessivas na microestrutura de um metal durante a deformação plástica. A primeira e mais óbvia é a mudança no formato dos grãos, que se tornam mais alongados, acompanhada de um considerável aumento na área total dos contornos de grão. Ao mesmo tempo discordâncias são geradas continuamente durante a deformação e passam a interagir entre si levando à formação de subestruturas mais

* Contribuição técnica ao 69 Congresso Anual da ABM - Internacional e ao 14ํㅡㄹ ENEMET - Encontro Nacional de Estudantes de Engenharia Metalúrgica, de Materiais e de Minas, 21 a 25 de julho de 2014, São Paulo, SP, Brasil. 


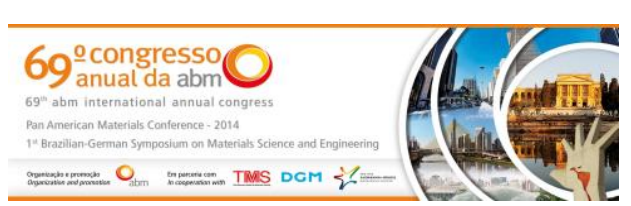

complexas. Essas subestruturas são formadas por arranjos de discordâncias que tendem a se agrupar em células ou subgrãos, a fim de acomodar as crescentes tensões geradas e também as crescentes diferenças de orientação entre cristais. A estrutura é de células de discordâncias, quando o contorno de baixo ângulo é difuso. E esta evolui para uma estrutura de subgrão quando o contorno se torna bem definido [6]. Comportamento semelhante já foi relatado por Iwahashi et al [7] ao analisarem o refinamento um alumínio de alta pureza deformado via ECAP através das rotas A e C. Observaram que após o primeiro passe obteve-se uma microestrutura homogênea constituída de bandas paralelas de subgrãos com formato alongado, separados por contornos de baixo ângulo. Com a repetição dos passes, esses contornos evoluíram para contornos de alto ângulo. A investigação da evolução microestrutural durante dois passes de deformação permitiu concluir que os principais mecanismos responsáveis pela redução do tamanho de grão foram a fragmentação e rotação dos grãos, de acordo com o modelo de refinamento de grãos proposto por Langdon [8], ainda que este modelo não relacione a formação de subgrãos. Os resultados encontrados neste trabalho referentes à evolução da microestrutura se confirmam quando comparados com aqueles existentes na literatura. Nesses trabalhos também foi observado que o tamanho do grão da amostra se reduziu drasticamente após o primeiro passe de deformação, seguido de um decréscimo gradual no segundo passe. Além do aumento da fração de contornos de alto ângulo também associado com o aumento do número de passes [3,9,10].

A avaliação da evolução da textura cristalográfica foi realizada através das funções distribuição de orientação, as ODFs, apresentadas na Figura 5, que representam os cortes nos quais foram alcançados níveis mais altos de intensidade, $f(g)$. A Tabela 2 fornece as orientações e intensidade das componentes de textura identificadas na liga.

(a)
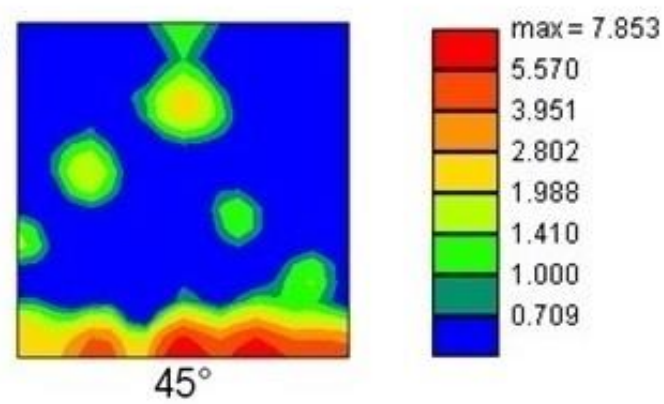

(c)

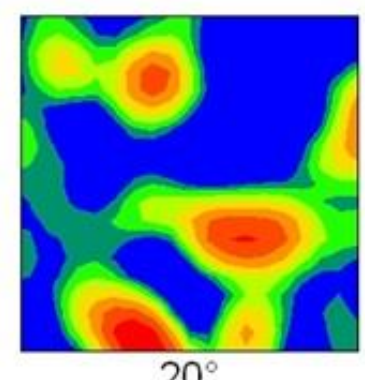

(b)

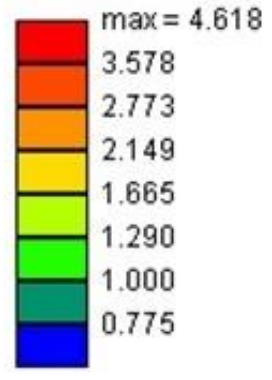

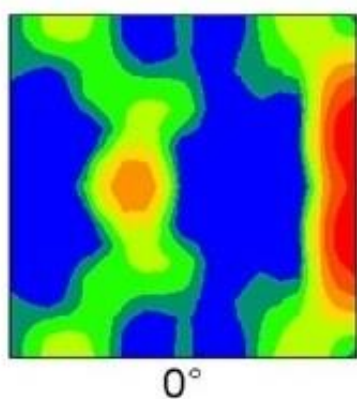
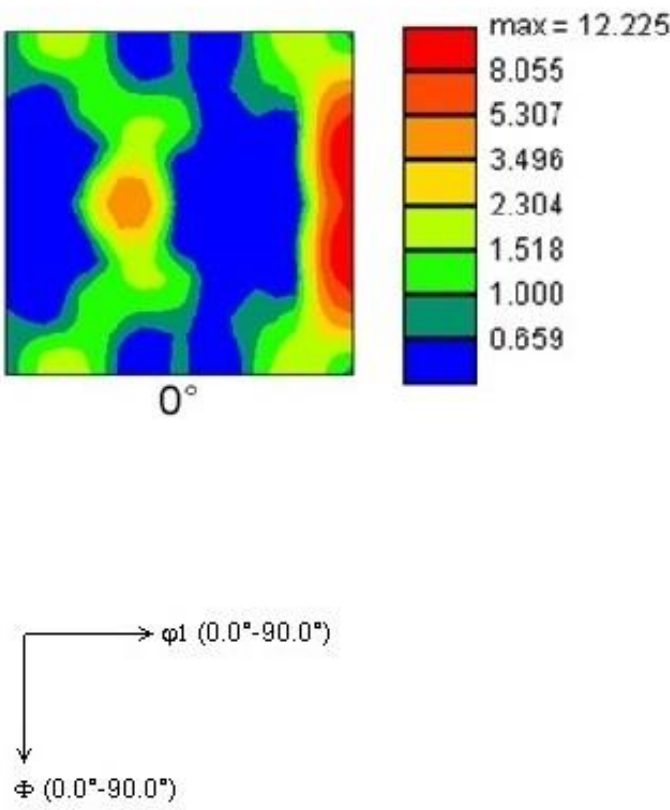

Figura 5: ODF do material deformado via ECAP: a) Material de partida; b) primeiro passe; c) segundo passe.

\footnotetext{
* Contribuição técnica ao $69^{\circ}$ Congresso Anual da ABM - Internacional e ao 14ํㅡㄹ ENEMET - Encontro Nacional de Estudantes de Engenharia Metalúrgica, de Materiais e de Minas, 21 a 25 de julho de 2014, São Paulo, SP, Brasil.
} 
(a)

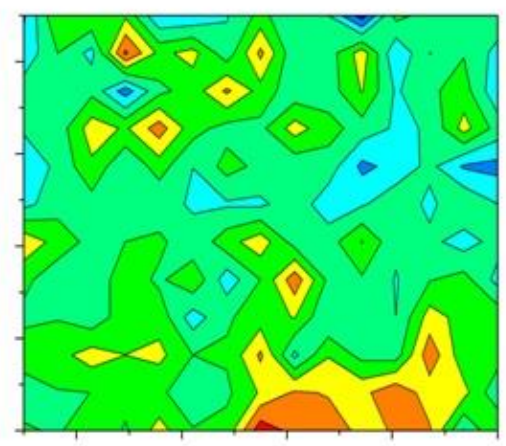

(b)

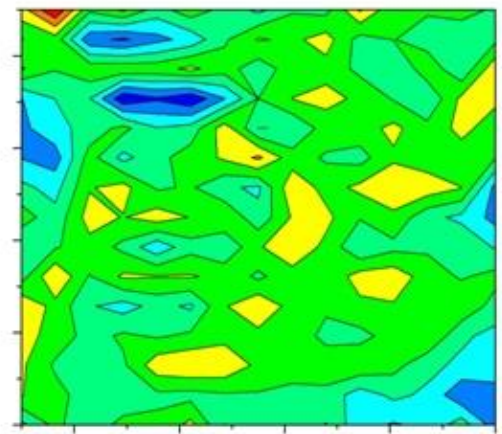

(c)
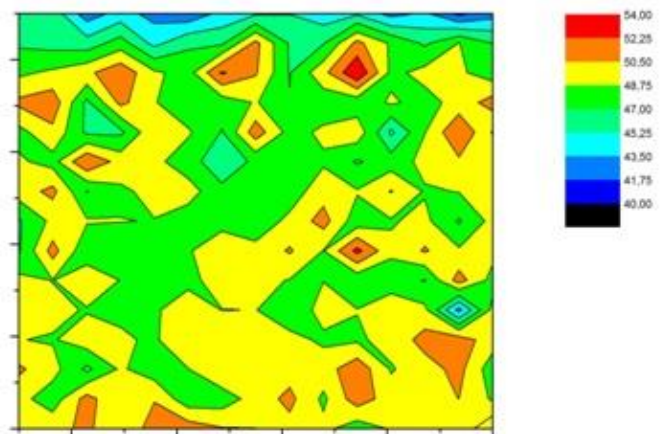

Figura 7: Mapas de dureza da liga Al AA 1070 deformada via ECAP. (a) Material de partida; (b) primeiro passe; (c) segundo passe.

Comportamento similar foi encontrado por Iwahashi e seus colaboradores em [7], que ao analisarem o comportamento de uma liga de alumínio de alta pureza deformada via ECAP através das rotas $\mathrm{A}$ e $\mathrm{C}$, notaram que ambas as rotas levam a um aumento significativo da dureza após o primeiro passe. Subsequentemente, a dureza aumentou levemente até o terceiro passe e depois houve um decréscimo gradual da mesma com os passes seguintes. Resultado semelhante também foi encontrado por Saravanan et al [12] ao investigar uma liga de alumínio com diferente nível de pureza, concluíram que o processo de ECAP leva a um aumento considerável na dureza após o primeiro passe e se mantém a uma taxa baixa até o sexto passe. Deste momento em diante apesar do aumento no número de passes os valores da dureza decrescem. O estado estacionário dos valores da dureza encontrado durante o processamento via ECAP é explicado pela ocorrência de recuperação dinâmica, uma vez que a recuperação permite que a quantidade de defeitos gerados durante a deformação seja compensada pela quantidade de defeitos eliminados pela movimentação das discordâncias [13].

\section{CONCLUSÃO}

Os resultados obtidos permitiram concluir que o material de partida, previamente laminado a quente, apresentou características dos fenômenos de restauração, o que facilitou a ocorrência do fenômeno de recuperação dinâmica observado no material deformado. Um intenso refinamento na microestrutura do material foi observado após os passes de deformação, permitindo a conclusão de que os principais mecanismos responsáveis pelo refinamento do grão foram os processos de subdivisão através da rotação e fragmentação dos grãos. Os resultados da análise

\footnotetext{
* Contribuição técnica ao 69ำ Congresso Anual da ABM - Internacional e ao 14ํㅡㄹ ENEMET - Encontro Nacional de Estudantes de Engenharia Metalúrgica, de Materiais e de Minas, 21 a 25 de julho de 2014, São Paulo, SP, Brasil.
} 
